Edith Cowan University

Research Online

Research outputs 2014 to 2021

3-3-2019

Cystatin C and risk of new-onset depressive symptoms among individuals with a normal creatinine-based estimated glomerular filtration rate: A prospective cohort study

Li Haibin

Wang Anxin

Qi Gao

Guo Jin

Li Xia

See next page for additional authors

Follow this and additional works at: https://ro.ecu.edu.au/ecuworkspost2013

Part of the Nephrology Commons

10.1016/j.psychres.2019.01.009

This is an Author's Accepted Manuscript of: Li, H., Wang, A., Qi, G., Guo, J., Li, X., Wang, W., ... Guo, X. (2019).

Cystatin $C$ and risk of new-onset depressive symptoms among individuals with a normal creatinine-based

estimated glomerular filtration rate: A prospective cohort study. Psychiatry Research, 273, 75-81. Available here

(C) 2019. This manuscript version is made Available under the CC-BY-NC-ND 4.0 license

http://creativecommons.org/licenses/by-nc-nd/4.0/

This Journal Article is posted at Research Online.

https://ro.ecu.edu.au/ecuworkspost2013/5630 
Authors

Li Haibin, Wang Anxin, Qi Gao, Guo Jin, Li Xia, Wei Wang, Xiang Yutao, and Guo Xiuhua

This journal article is available at Research Online: https://ro.ecu.edu.au/ecuworkspost2013/5630 
(C) 2019. This manuscript version is made available under the CC-BY-NC-ND 4.0 license http://creativecommons.org/licenses/by-nc-nd/4.0/

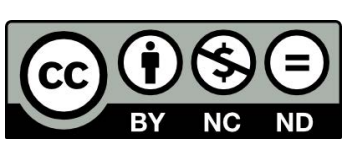




\section{Cystatin C and Risk of New-Onset Depressive Symptoms Among Individuals with a Normal Creatinine-Based Estimated Glomerular Filtration Rate: A Prospective Cohort Study \\ Running title: Cystatin $\mathrm{C}$ and Depressive Symptoms}

\section{Authors:}

Haibin $\mathrm{Li}^{\mathrm{a}, \mathrm{b}}$; Anxin Wang ${ }^{\mathrm{c}}$; Gao $\mathrm{Qi}^{\mathrm{a}}$, ; Jin Guo ${ }^{\mathrm{d}}$; Xia Li ${ }^{\mathrm{e}}$; Wei Wang ; Yutao Xiangg; Xiuhua Guo ${ }^{\mathrm{a}, \mathrm{b}}$

${ }^{\text {a}}$ Department of Epidemiology and Health Statistics, School of Public Health, Capital Medical University, Beijing, China.

${ }^{b}$ Beijing Municipal Key Laboratory of Clinical Epidemiology, Capital Medical University, Beijing, China.

'Department of Neurosurgery, Beijing Tiantan Hospital, Capital Medical University, Beijing, China.

${ }^{\mathrm{d}}$ Guanghua Group Pty Ltd, Melbourne, Victoria, Australia.

${ }^{\text {eDepartment }}$ of Mathematics and Statistics, La Trobe University, Melbourne, Victoria, Australia.

${ }^{\mathrm{f}}$ Global Health and Genomics, School of Medical Sciences and Health, Edith Cowan University, Perth, Western Australia, Australia.

${ }^{g}$ Unit of Psychiatry, Faculty of Health Sciences, University of Macau, Macao SAR, China.

\section{*Corresponding Author:}

Xiuhua Guo

Address: School of Public Health, Capital Medical University, No.10 Xitoutiao, You'anmen Wai, Fengtai District, Beijing 100069, China

Cellular: +86-010-83911508

E-mail: statguo@ccmu.edu.cn 


\section{Introduction}

Chronic kidney disease (CKD) or reduced renal function with advanced age is highly prevalent in the elderly ( function is associated with cardiovascular diseases (Ix, Shlipak, Chertow and Whooley, 2007; Matsushita et al., 2012), all-cause mortality (Shlipak et al., 2005) and cognitive decline (Buchman et al., 2009) in the elderly. Generally, the glomerular filtration rate (GFR) estimated from the serum creatinine is considered the best overall index of renal function in the clinical practice (Traynor, Mactier, Geddes and Fox, 2006). However, serum creatinine levels are influenced by age, gender, body weight and muscle mass (Stevens, Coresh, Greene and Levey, 2006). Consequently, cystatin C has been identified as a novel alternative endogenous marker of renal function and is more sensitive than creatinine (Rule, Bailey, Lieske, Peyser and Turner, 2013; Shardlow et al., 2017). Previous studies suggest that the cystatin $\mathrm{C}$ level has a stronger association with cardiovascular disease and mortality than do serum creatinine and GFR in the elderly (Shlipak et al., 2005). Blood urea nitrogen (BUN) is a serum byproduct of protein metabolism and is used to evaluate renal function (Macedo, 2011). The BUN concentration rises as renal function decreases; however, BUN may vary independently of the GFR (Lopez-Giacoman and Madero, 2015).

Depressive symptoms are a major global public health problem. Depression is also highly common among the elderly and is linked to a wide range of adverse health outcomes including cardiovascular disease and mortality (Ye et al., 2013). It is estimated that approximately $26.5 \%$ of individuals with CKD experience depressive symptoms, and this number rises to $40 \%$ for the subset of individuals who require dialysis treatment (Palmer et al., 2013). Participants with kidney impairment suffer severe psychological distress (Tong et al., 2009), and they have a 3-fold higher risk of depressive symptoms than individuals without kidney impairment (Palmer et al., 2013). Additionally, depressive symptoms increase the risk of end-stage renal disease by $66 \%$ (Tsai et al., 2012). The majority of previous studies have focused on the impact of depressive symptoms on the risk of CKD or death (Ix, Shlipak, Chertow and Whooley, 2007; Shlipak et 
al., 2005; Tsai et al., 2012; Wu et al., 2010); however, research on the relationship between renal function and depressive symptoms in the general population are scarce and conflicting. For example, Odden et al. conducted a cross-sectional study of 967 patients with cardiovascular disease who were enrolled in the Heart and Soul Study; this study showed that impaired renal function, as indicated by creatinine clearance, was not associated with depressive symptoms (Odden, Whooley and Shlipak, 2006). In the Maastricht Study, high albuminuria levels were cross-sectionally associated with depressive symptoms (Martens et al., 2016). In addition, in the Health, Aging and Body Composition Study of 3075 subjects aged 70-79 years over 6 years of follow up, Minev et al. found that a high cystatin $\mathrm{C}(>1.25 \mathrm{mg} / \mathrm{l})$ was associated with a 2 -fold increased risk of depressive symptoms (Minev et al., 2010). However, this association has not been adequately evaluated among older adults with normal renal function.

To the best of our knowledge, no study has compared the effects of various renal function measurements on the risk of depressive symptoms in a middle-aged adult with normal renal function. Therefore, we aimed to examine the associations of cystatin $\mathrm{C}$, BUN and the estimated GFR with the risk of depressive symptoms in a populationbased cohort study of middle-aged Chinese adults. We hypothesized that cystatin C would be a stronger and linear predictor of the risk of depressive symptoms among subjects with normal function than the BUN or GFR.

\section{Methods}

\subsection{Study Participants}

The China Health and Retirement Longitudinal Study (CHARLS) is a communitybased, national representative longitudinal study that was initiated in 2011-2012 (Zhao, $\underline{\mathrm{Hu}}$, Smith, Strauss, and Yang, 2014). The main objective of CHARLS is to evaluate the main health and economic issues associated with rapid population aging in China. Briefly, 17708 individuals aged 45 years or older were randomly recruited from 150 counties or districts from 28 provinces using a four-stage, stratified, and cluster sampling method. Among those participants, 11847 individuals provided fasting blood 
samples. A description of the study design, quality control procedures, laboratory methods, and procedures for physical examination can be found on the CHARLS website (http://charls.pku.edu.cn/en). The ethical committees of Peking University approved the study protocol, and all participants provided written informed consent. For this analysis, 5111 participants without depressive symptoms, whose serum was available for the measurement of cystatin C, and who had a normal renal function (GFR $\geq 60 \mathrm{ml} / \mathrm{min} / 1.73 \mathrm{~m}^{2}$ ) were included. The cohort selection of flowchart is included in the Supplementary Materials (Figure S1).

\subsection{Measurements of Renal Function}

Serum from fasting blood samples was stored at $-70^{\circ} \mathrm{C}$ until analysis at the Center for Clinical Laboratory of Capital Medical University. Cystatin C was measured using a particle-enhanced turbidimetric assay (Voskoboev, Larson, Rule and Lieske, 2012). The intra-individual coefficient of variation $(\mathrm{CV})$ was $<5 \%$, and the between-individual $\mathrm{CV}$ was $<5 \%$. The detection range of the assay was 0.5 to $8.0 \mathrm{mg}$ per liter. The serum creatinine level was measured using a rate-blanked and compensated Jaffe creatinine method. The intra-individual $\mathrm{CV}$ was $<1.6 \%$, and the between-individual $\mathrm{CV}$ was $<2.1 \%$. The detection range of the assay was 0.1 to $25 \mathrm{mg} / \mathrm{dL}$. We estimated the GFR using the CKD-EPI (Chronic Kidney Disease Epidemiology Collaboration) creatinine equation (Levey et al., 2009). The BUN was measured using an enzymatic UV method with urease. The intra-individual $\mathrm{CV}$ was $<4.4 \%$, and the between-individual $\mathrm{CV}$ was $<4.1 \%$. The range of detection of the assay was 5 to $100 \mathrm{mg} / \mathrm{dL}$.

\subsection{Ascertainment of Depressive Symptoms}

Depressive symptoms were evaluated at baseline and every two years at follow-up using the 10-item Center for Epidemiologic Studies Depressive Symptoms Scale (CESD) short form (Andresen, Malmgren, Carter and Patrick, 1994). To clarify frequency of the mood and behavioral symptoms, participants were asked to rate how often they experienced then symptoms during the previous week: (1) I was bothered by things that do not usually bother me; (2) I had trouble keeping my mind on what I was doing; (3) 
I felt depressed; (4) I felt everything I did was an effort; (5) I felt hopeful about the future; (6) I felt fearful; (7) My sleep was restless; (8) I was happy; (9) I felt lonely; (10) I could not get "going". Each item was scored on a 4-point Likert scale ranging from 0 ("not at all") to 3 ("nearly every day"). Items 5 and 8 were reversely scored before analysis. In the current analysis, individuals missing three or more responses on the CES-D were excluded. Therefore, the total CES-D scores ranged from 0 to 30 , with a higher score representing a greater level of depressive symptoms. The 10-item CES-D has been widely validated among older Chinese adults and shows satisfactory psychometric properties (Boey, 1999; Chen and Mui, 2014; Cheng and Chan, 2005). A recent validation study conducted in a subsample of 742 CHARLS participants aged $\geq 60$ years showed that the two-factor model was the best fit, and the completely standardized factor loadings were $\geq 0.30$ (Chen and Mui, 2014). Following the validation study, the binary depressive symptoms were defined as total CES-D scores of 12 or higher in the current study (Chen and Mui, 2014). We excluded those individuals who had depressive symptoms at baseline.

\subsection{Assessment of Covariates}

Baseline demographic and risk factors were collected by trained personnel according to standard procedures. Sociodemographic factors, including age, sex, residential area (urban, rural), education level (no formal education, primary school, middle school or above), marital status (unmarried, married) and retirement status (retired, nonretired) were self-reported. Health behaviors including smoking (never, former, current), alcohol use (never, $<1$ times/month, $\geq 1$ times/month) were assessed using a questionnaire. Self-reported health was recoded into three categories (very good/good, fair, poor/very poor). Life satisfaction was recoded into three categories (completely/very satisfied, somewhat satisfied, not very/not at all satisfied). Familybased social support was assessed by evaluating the family-based network size measured by the number of core family members, whether the respondent had received help and whether the respondent had provided help (Shen and Yeatts, 2013). Social participation was assessed by respondent participation in the following social activities: 
(1) interacted with a friend; (2) played mahjong, or went to a sport, social or other kind of club; (3) took part in a community-related organization; (4) took part in a community-related organization; (5) took part in voluntary or charity work; (6) attended

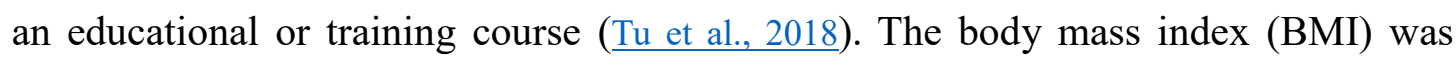
calculated as the weight in kilograms divided by the height in meters squared. Sitting blood pressure was measured three times after a 5-min rest period in quiet space using an Omron ${ }^{\mathrm{TM}}$ HEM-7200 Monitor, and an average of three values was obtained for the systolic blood pressure (SBP) and diastolic blood pressure (DBP). The self-reported prior history of physician-diagnosed diseases included hypertension, diabetes mellitus, dyslipidemia, coronary artery heart disease, stroke, and obstructive pulmonary disease. Using plasma samples, the total cholesterol (TC), low-density lipoprotein cholesterol (LDL-C), high-density lipoprotein cholesterol (HDL-C), triglycerides (TG), fasting blood glucose (FBG), and C-reactive protein (CRP) levels were measured using standard assays (Yang et al., 2016).

\subsection{Statistical Analysis}

All statistical analyses were performed using Stata/MP, version 14.1, and 2-sided $P$ values $<0.05$ were considered statistically significant. In the cohorts, the data were $80 \%$ complete in relation to potential confounders. We first used multiple imputation with multivariable chained equations to impute missing data for continuous variables (BMI, SBP, DBP, HDL-C, LDL-C, and FBG) and categorical variables (retirement status, receiving help, providing help, self-reported health and life satisfaction) (Harel and Zhou, 2007). Because family-network size, TG and CRP values were not normally distributed, $\log$ transformation was used. Participants were divided into five groups based on the quintiles of cystatin $\mathrm{C}$ levels based on values for the entire cohort $(<0.82 \mathrm{mg} / 1,0.83$ $0.93 \mathrm{mg} / 1,0.94-1.02 \mathrm{mg} / 1,1.03-1.16 \mathrm{mg} / 1$, and $\geq 1.17 \mathrm{mg} / \mathrm{l})$. The $P$ value for linear trend across the quintiles was calculated from the logistic regression for categorical variables and linear regression for continuous variables.

To evaluate the association of each renal function measurement with depressive 
symptoms, we initially created quintiles of the study population according to the cystatin C, BUN and estimated GFR levels. We used a modified Poisson regression with robust error variance (McNutt, $\mathrm{Wu}, \mathrm{Xue}$ and Hafner, 2003) to evaluate the likelihood of developing depressive symptoms (yes or no) at follow-up for each index of renal function among participants who were free of depressive symptoms at baseline. Incidence risk ratio (IRR) and the corresponding 95\% confidence interval (CI) were reported. The main analysis was based on the combined the results obtained by running each model in 10 independent imputed data sets. Potential confounding variables for statistical adjustment were chosen a priori according to known associations with depressive symptoms and renal function. The analysis was adjusted for age, sex, education, marital status, living area, alcohol use, smoking status, retirement status, social participation, family-based social support, self-reported health, life satisfaction, history of hypertension, diabetes, dyslipidemia, coronary artery heart disease, stroke, and chronic obstructive pulmonary disorder, SBP, BMI, TC, LDL-C, HDL-C, baseline CES-D score and log-transformed TG, and log-transformed CRP level. For tests of linear trend, we applied linear terms using the median of each renal function measurement quintile. The association of cystatin $\mathrm{C}, \mathrm{BUN}$ or the estimated GFR with depressive symptoms were also assessed as a continuous scale (per 1-SD change).

Furthermore, the association between incident depressive symptoms and cystatin $\mathrm{C}$ levels on a continuous scale was examined using restricted cubic splines with 3 knots located at the 10th, 50th, and 90th percentiles ( in a modified Poisson regression. A cystatin $C$ concentration of $1 \mathrm{mg} / \mathrm{l}$ was used as the reference value for this analysis because it approximated the median value. Sensitivity analysis using complete data $(\mathrm{n}=4128)$ was conducted to examine the association between measurements of renal function and depressive symptoms.

\section{Results}

\subsection{Study Participants}


Among the 5111 individuals included in this analysis, the overall mean $( \pm \mathrm{SD})$ serum cystatin $\mathrm{C}$ was $0.99 \pm 0.21 \mathrm{mg} / \mathrm{l}$ (1st to 99 th percentile range, 0.55 to $1.58 \mathrm{mg} / \mathrm{l})$. The baseline characteristics of the participants are shown in Table 1. The participants with the highest cystatin $\mathrm{C}$ levels were more likely to be older, male, and current smokers and drinkers, compared with participants with lower cystatin C levels (Table 1). Nearly all of the coexisting conditions were more prevalent among those with elevated levels of cystatin C (Table 1).

\subsection{Incidence Rate of Depressive symptoms}

During 4 years of follow-up, 1746 (34.16\%) individuals developed new-onset depressive symptoms. The crude incidence rate of depressive symptoms across each quintile of cystatin C, BUN, and estimated GFR are shown in Figure 1.

\subsection{Renal Function with Depressive symptoms}

Table 2 shows the IRR and $95 \% \mathrm{CI}$ of depressive symptoms according to measures of renal function. After multivariate adjustment, compared to those with the lowest level of cystatin C (Quintile 1), individuals with the highest level of cystatin C (Quintile 5) had an adjusted IRR of 1.18 (95\% CI: 1.03-1.35) for depressive symptoms. Individuals with an intermediate BUN level (Quintile 3) were at lower risk of depressive symptoms (IRR: 0.86, 95\% CI: 0.76-0.97). Individuals with lower estimated GFR values were showed an at increased depressive symptoms risk (IRR: 1.15, 95\% CI: 1.01-1.31). The association remained robust when analysis limited to complete data (Table S1).

\subsection{Dose-Response Analysis}

The restricted cubic spline analysis suggested a linear relationship between cystatin C and depressive symptoms (Figure 2). The multivariate-adjusted IRR using serum cystatin C levels on a continuous scale was 1.13 (95\% CI: 1.08-1.19) per 1-SD change (Figure 3). The association between the BUN and estimated GFR on a continuous scale and depressive symptoms were not statistically significantly (Figure 3). 


\section{Discussion}

Our results demonstrate that the cystatin C concentration is linearly and independently associated with new-onset depressive symptoms in a general population with a creatinine-based estimated GFR of $60 \mathrm{~mL} / \mathrm{min} / 1.73 \mathrm{~m}^{2}$ or greater. In contrast, the BUN level and estimated GFR on a continuous scale had no statistically significant association with depressive symptoms in the fully adjusted model.

\subsection{Association between Renal Function and Depressive Symptoms}

Depressive symptoms are a major contributor to the global disease burden and are highly prevalent in patients with chronic kidney disease or end-stage renal disease. A recent meta-analysis reported that the prevalence of depressive symptoms was as high as 40\% among CKD patients (Palmer et al., 2013). Several previous studies suggested that impaired renal function measured by estimated GFR may be associated with depressive symptoms (Abdel-Kader, Unruh and Weisbord, 2009; Fischer et al., 2012; Liu et al., 2018), whereas other studies did not find this association (Campbell et al., 2013; Fischer et al., 2010; Martens et al., 2016). For example, in the Chronic Renal Insufficiency Cohort (CRIC) Study, Fischer et al. found that there were an $8 \%$ increased odds of depressive symptoms for every $10 \mathrm{ml} / \mathrm{min} / 1.73 \mathrm{~m}^{2}$ decrease in GFR in the fully adjusted model (Fischer et al., 2012). This association was also observed in an older Chinese crosssectional study (Liu et al., 2018). Furthermore, a recent meta-analysis conducted by Lee et al. demonstrated that self-management programs for the estimated GFR improved depressive symptoms in CKD patients (Lee, Wu, Hsieh and Tsai, 2016). Additionally, in the Diabetes Study of Northern California survey, older adults in the lowest estimated GFR category $\left(\leq 29 \mathrm{~mL} / \mathrm{min} / 1.73 \mathrm{~m}^{2}\right)$ were cross-sectionally demonstrated to have twice the probability of depressive symptoms compared with these with the normal estimated GFR category $\geq 90 \mathrm{~mL} / \mathrm{min} / 1.73 \mathrm{~m}^{2}$ ) (Campbell et al., 2013). However, this association was no longer statistically significant adjustment for the hemoglobin level (Campbell et al., 2013). In the current analysis, we did not find a perspective significantly association between the estimated GFR and depressive symptoms. Our finding was consistent with previous studies (Campbell et al., 2013; Martens et al., 2016). 
Previous studies on the association of cystatin $\mathrm{C}$ with depressive symptoms are lacking. One study conducted by Minev et al. reported that a high level of cystatin C ( $>1.25 \mathrm{mg} / \mathrm{l})$ increased the risk of depressive symptoms 2-fold during a 6-year follow-up in the American healthy elders (Minev et al., 2010). In addition, the Confucius Hometown Aging Project showed that a high serum cystatin $\mathrm{C}$ level and impaired kidney function were longitudinally associated with incident geriatric depressive symptoms among Chinese older adults (Wu et al., 2018). However, that study did not compare the relative association of cystatin C, BUN and the estimated GFR with the risk of new-onset depressive symptoms. Our results were robust to statistical adjustment of a series of confounders. Our results provide the new evidence that cystatin $\mathrm{C}$ increases the risk of depressive symptoms in a middle-aged population. Further research is needed to validate our findings regarding the association between renal function and depressive symptoms; this relationship has not been thoroughly explored among the elderly.

Cystatin $\mathrm{C}$ as a measure of renal function overcomes the limitations of using the creatinine level because $\mathrm{C}$ concentration is not influenced by age, sex, or body mass (Fliser and Ritz, 2001). We also used restricted cubic splines to estimate the trend in the risk for depressive symptoms. The cystatin $\mathrm{C}$ concentration was confirmed to have a linear relationship with the risk of depressive symptoms. These findings were in alignment with prior studies that reported a linear relationship between cystatin $\mathrm{C}$ and an increased the risk of heart failure ( $\underline{\text { Sarnak et al., 2005), hypertension (Kestenbaum et al., }}$ 2008), cardiovascular disease and mortality (

Several potential mechanisms may explain the association between cystatin $\mathrm{C}$ and depressive symptoms. First, cystatin $\mathrm{C}$ was used as an index of cerebral small vessel disease (Wada et al., 2010), which has been associated with incident depressive symptoms (van Sloten et al., 2015); this relationship is termed the "vascular depressive symptoms hypothesis (Alexopoulos, 2006)." In addition, physiological factors such as endothelial dysfunction and inflammation, have both linked to cystatin C (Balta et al., 2013; Zhang et 
al., 2016) and depressive symptoms (Al Shweiki et al., 2017; Bouzinova et al., 2014; Miller and Raison, 2016).

\subsection{Limitations}

There were several limitations. First, depressive symptoms measured by CES-D are not equivalent to a clinical diagnosis of major depressive symptoms. Second, depressive symptoms tend to fluctuate over time (White et al., 2016), and we did not assess the variability in depressive symptoms. Additionally, owing to the observational nature of our investigation, unmeasured potential confounding factors such as physical activity may still be present. Several important strengths of this study were the prospective design, and the large sample size and the use of the validated CES-D to assess depressive symptoms in 28 provinces in China. An additional strength is the complete assessment of renal function, which included cystatin C, BUN, and estimated GFR. To the best of our knowledge, this study is first to explore the association between cystatin $\mathrm{C}$ and depressive symptoms in a large cohort with normal renal function.

\subsection{Conclusions}

Our study demonstrated that cystatin $\mathrm{C}$, a sensitive measure of renal function, is significantly associated with future depressive symptoms in the general population with relatively normal renal function. It is clinically important for individuals with normal renal function but high cystatin $\mathrm{C}$ to treat depressive symptoms and improve the quality of life. 


\section{Acknowledgments}

The China Health and Retirement Longitudinal Study was developed by a team of researchers based at Peking University. The funding is provided by the National Institute on Aging (grant numbers: 1-R21-AG031372-01, 1-R21-AG033675-01-A1, 1R01-AG037031-01 and 1-R01-AG037031-03S1), the National Natural Science Foundation of China (grant numbers: 70773002, 70910107022 and 71130002), the World Bank (grant numbers: 7145915 and 7159234), and Peking University. We sincerely thank those who participated in data collection and management.

\section{Sources of Funding}

This work was supported by The Program of Natural Science Fund of China (grant numbers: 81530087$)$.

\section{Declarations}

The authors declare that there is no duality of interest associated with this manuscript. 


\section{References:}

Abdel-Kader, K., Unruh, M.L., Weisbord, S.D., 2009. Symptom burden, depression, and quality of life in chronic and end-stage kidney disease. Clin J Am Soc Nephro 4 (6), 1057-1064.

Al Shweiki, M.H.D.R., Oeckl, P., Steinacker, P., Hengerer, B., Schonfeldt-Lecuona, C., Otto, M., 2017. Major depressive disorder: insight into candidate cerebrospinal fluid protein biomarkers from proteomics studies. Expert Rev Proteomic 14 (6), 499-514.

Alexopoulos, G.S., 2006. The vascular depression hypothesis: 10 years later. Biol Psychiat 60 (12), $1304-$ 1305.

Andresen, E.M., Malmgren, J.A., Carter, W.B., Patrick, D.L., 1994. Screening for depression in well older adults - evaluation of a short-form of the CES-D (Center for Epidemiologic Studies Depression Scale). Am J Prev Med 10 (2), 77-84.

Balta, S., Demirkol, S., Ay, S.A., Cakar, M., Sarlak, H., Celik, T., 2013. Serum cystatin-c levels correlate with endothelial dysfunction in patients with the metabolic syndrome. J Intern Med 274 (2), 200-201.

Boey, K.W., 1999. Cross-validation of a short form of the CES-D in chinese elderly. Int J Geriatr Psychiatry 14 (8), 608-617.

Bouzinova, E.V., Norregaard, R., Boedtkjer, D.M.B., Razgovorova, I.A., Moeller, A.M.J., Kudryavtseva, O., et al., 2014. Association between endothelial dysfunction and depression-like symptoms in chronic mild stress model of depression. Psychosom Med 76 (4), 268-276.

Buchman, A.S., Tanne, D., Boyle, P.A., Shah, R.C., Leurgans, S.E., Bennett, D.A., 2009. Kidney function is associated with the rate of cognitive decline in the elderly. Neurology 73 (12), 920-927.

Campbell, K.H., Huang, E.S., Dale, W., Parker, M.M., John, P.M., Young, B.A., et al., 2013. Association between estimated GFR, health-related quality of life, and depression among older adults with diabetes: the Diabetes and Aging Study. Am J Kidney Dis 62 (3), 541-548.

Chen, H., Mui, A.C., 2014. Factorial validity of the center for epidemiologic studies depression scale short form in older population in China. Int Psychogeriatr 26 (1), 49-57.

Cheng, S.T., Chan, A.C., 2005. The center for epidemiologic studies depression scale in older chinese: thresholds for long and short forms. Int J Geriatr Psychiatry 20 (5), 465-470.

Fischer, M.J., Kimmel, P.L., Greene, T., Gassman, J.J., Wang, X.L., Brooks, D.H., et al., 2010. Sociodemographic factors contribute to the depressive affect among african americans with chronic kidney disease. Kidney Int 77 (11), 1010-1019.

Fischer, M.J., Xie, D.W., Jordan, N., Kop, W.J., Krousel-Wood, M., Tamura, M.K., et al., 2012. Factors associated with depressive symptoms and use of antidepressant medications among participants in the chronic renal insufficiency cohort (CRIC) and hispanic-CRIC studies. Am J Kidney Dis 60 (1), 27-38.

Fliser, D., Ritz, E., 2001. Serum cystatin c concentration as a marker of renal dysfunction in the elderly. Am J Kidney Dis 37 (1), 79-83.

Harel, O., Zhou, X.H., 2007. Multiple imputation: review of theory, implementation and software. Stat Med 26 (16), 3057-3077.

Harrell, Frank E, 2010. Regression modeling strategies: with applications to linear models, logistic regression, and survival analysis. Springer.

Hill, N.R., Fatoba, S.T., Oke, J.L., Hirst, J.A., O'Callaghan, C.A., Lasserson, D.S., et al., 2016. Global prevalence of chronic kidney disease - a systematic review and meta-analysis. Plos One 11 (7), 
e0158765.

Ix, J.H., Shlipak, M.G., Chertow, G.M., Whooley, M.A., 2007. Association of cystatin c with mortality, cardiovascular events, and incident heart failure among persons with coronary heart disease data from the Heart and Soul Study. Circulation 115 (2), 173-179.

Kestenbaum, B., Rudser, K.D., de Boer, I.H., Peralta, C.A., Fried, L.F., Shlipak, M.G., et al., 2008. Differences in kidney function and incident hypertension: the Multi-Ethnic Study of Atherosclerosis. Annals of Internal Medicine 148 (7), 501-508.

Lee, M.C., Wu, S.F.V., Hsieh, N.C., Tsai, J.M., 2016. Self-management programs on eGFR, depression, and quality of life among patients with chronic kidney disease: a meta-analysis. Asian Nurs Res $10(4), 255-262$.

Levey, A.S., Stevens, L.A., Schmid, C.H., Zhang, Y.L., Castro, A.F., 3rd, Feldman, H.I., et al., 2009. A new equation to estimate glomerular filtration rate. Ann Intern Med 150 (9), 604-612.

Liu, Q., Li, Y.X., Hu, Z.H., Jiang, X.Y., Li, S.J., Wang, X.F.J.N.L., 2018. Reduced estimated glomerular filtration rate is associated with depressive symptoms in elder chinese: a population-based crosssectional study. Neurosci Lett. 666, 127-132.

Lopez-Giacoman, S., Madero, M., 2015. Biomarkers in chronic kidney disease, from kidney function to kidney damage. World J Nephrol 4 (1), 57-73.

Macedo, E., 2011. Blood urea nitrogen beyond estimation of renal function. Crit Care Med 39 (2), 405 406.

Martens, R.J.H., Kooman, J.P., Stehouwer, C.D.A., Dagnelie, P.C., Kallen, C.J.H.V.D., Kroon, A.A., et al., 2016. Albuminuria is associated with a higher prevalence of depression in a populationbased cohort study: the Maastricht Study. Nephrol Dial Transplant 33(1), 128-138.

Matsushita, K., Mahmoodi, B.K., Woodward, M., Emberson, J.R., Jafar, T.H., Jee, S.H., et al., 2012. Comparison of risk prediction using the CKD-EPI equation and the mdrd study equation for estimated glomerular filtration rate. JAMA 307 (18), 1941-1951.

McNutt, L.A., Wu, C., Xue, X., Hafner, J.P., 2003. Estimating the relative risk in cohort studies and clinical trials of common outcomes. Am J Epidemiol 157 (10), 940-943.

Miller, A.H., Raison, C.L., 2016. The role of inflammation in depression: from evolutionary imperative to modern treatment target. Nat Rev Immunol 16 (1), 22-34.

Minev, E., Unruh, M., Shlipak, M.G., Simsonick, E., Yaffe, K., Leak, T.S., et al., 2010. Association of cystatin $\mathrm{c}$ and depression in healthy elders: the Health, Aging and Body Composition Study. Nephron Clinical Practice 116 (3), C241-C246.

Odden, M.C., Whooley, M.A., Shlipak, M.G., 2006. Depression, stress, and quality of life in persons with chronic kidney disease: the Heart and Soul Study. Nephron Clin Pract 103 (1), c1-7.

Palmer, S., Vecchio, M., Craig, J.C., Tonelli, M., Johnson, D.W., Nicolucci, A., et al., 2013. Prevalence of depression in chronic kidney disease: systematic review and meta-analysis of observational studies. Kidney Int 84 (1), 179-191.

Rule, A.D., Bailey, K.R., Lieske, J.C., Peyser, P.A., Turner, S.T., 2013. Estimating the glomerular filtration rate from serum creatinine is better than from cystatin $\mathrm{c}$ for evaluating risk factors associated with chronic kidney disease. Kidney Int 83 (6), 1169-1176.

Sarnak, M.J., Katz, R., Stehman-Breen, C.O., Fried, L.F., Jenny, N.S., Psaty, B.M., et al., 2005. Cystatin c concentration as a risk factor for heart failure in older adults. Ann Intern Med. 142 (7), $497-$ 505.

Shardlow, A., McIntyre, N.J., Fraser, S.D.S., Roderick, P., Raftery, J., Fluck, R.J., et al., 2017. The clinical 
utility and cost impact of cystatin c measurement in the diagnosis and management of chronic kidney disease: a primary care cohort study. Plos Med 14 (10), e1002400.

Shen, Y.Y., Yeatts, D.E., 2013. Social support and life satisfaction among older adults in china: familybased support versus community-based support. Int J Aging Hum Dev 77 (3), 189-209.

Shlipak, M.G., Sarnak, M.J., Katz, R., Fried, L.F., Seliger, S.L., Newman, A.B., et al., 2005. Cystatin c and the risk of death and cardiovascular events among elderly persons. New Engl J Med 352 (20), 2049-2060.

Stevens, L.A., Coresh, J., Greene, T., Levey, A.S., 2006. Medical progress - assessing kidney function measured and estimated glomerular filtration rate. New Engl J Med 354 (23), 2473-2483.

Tong, A., Sainsbury, P., Chadban, S., Walker, R.G., Harris, D.C., Carter, S.M., et al., 2009. Patients' experiences and perspectives of living with ckd. Am J Kidney Dis 53 (4), 689-700.

Traynor, J., Mactier, R., Geddes, C.C., Fox, J.G., 2006. How to measure renal function in clinical practice. BMJ 333 (7571), 733-737.

Tsai, Y.C., Chiu, Y.W., Hung, C.C., Hwang, S.J., Tsai, J.C., Wang, S.L., et al., 2012. Association of symptoms of depression with progression of ckd. Am J Kidney Dis 60 (1), 54-61.

Tu, R.P., Inoue, Y., Yazawa, A., Hao, X.N., Cai, G.X., Li, Y.P., et al., 2018. Social participation and the onset of hypertension among the middle-aged and older population: evidence from the China Health and Retirement Longitudinal Study. Geriatr Gerontol Int 18 (7), 1093-1099.

van Sloten, T.T., Sigurdsson, S., van Buchem, M.A., Phillips, C.L., Jonsson, P.V., Ding, J., et al., 2015. Cerebral small vessel disease and association with higher incidence of depressive symptoms in a general elderly population: the AGES-Reykjavik Study. Am J Psychiat 172 (6), 570-578.

Voskoboev, N.V., Larson, T.S., Rule, A.D., Lieske, J.C., 2012. Analytic and clinical validation of a standardized cystatin c particle enhanced turbidimetric assay (PETIA) to estimate glomerular filtration rate. Clin Chem Lab Med 50 (9), 1591-1596.

Wada, M., Nagasawa, H., Kawanami, T., Kurita, K., Daimon, M., Kubota, I., et al., 2010. Cystatin c as an index of cerebral small vessel disease: results of a cross-sectional study in community-based japanese elderly. Eur J Neurol 17 (3), 383-390.

White, J., Zaninotto, P., Walters, K., Kivimaki, M., Demakakos, P., Biddulph, J., et al., 2016. Duration of depressive symptoms and mortality risk: the English Longitudinal Study of Ageing (ELSA). Br J Psychiat 208 (4), 337-342.

Wu, C.K., Lin, J.W., Caffrey, J.L., Chang, M.H., Hwang, J.J., Lin, Y.S., 2010. Cystatin c and long-term mortality among subjects with normal creatinine-based estimated glomerular filtration rates. J Am Coll Cardiol 56 (23), 1930-1936.

Wu, L., Yan, Z., Jiang, H., Xing, H., Li, H., Qiu, C.J.B.g., 2018. Serum cystatin c, impaired kidney function, and geriatric depressive symptoms among older people living in a rural area: a population-based study. BMC Geriatr 18 (1), 265.

Yang, F., Qian, D., Hu, D., Hou, M., Chen, S., Wang, P., et al., 2016. Prevalence of cardiovascular disease risk factor clustering in Chinese adults. Clinical Trials and Regulatory Science in Cardiology 15 (C), 1-6.

Ye, S., Muntner, P., Shimbo, D., Judd, S.E., Richman, J., Davidson, K.W., et al., 2013. Behavioral mechanisms, elevated depressive symptoms, and the risk for myocardial infarction or death in individuals with coronary heart disease: the REGARDS (reason for geographic and racial differences in stroke) study. J Am Coll Cardiol 61 (6), 622-630.

Zhang, M., Li, Y.L., Yang, X., Shan, H., Zhang, Q.H., Ming, Z.J., et al., 2016. Serum cystatin c as an 
inflammatory marker in exacerbated and convalescent copd patients. Inflammation 39 (2), 625 631.

Zhao, Y., Hu, Y., Smith, J.P., Strauss, J., Yang, G., 2014. Cohort profile: the China Health and Retirement Longitudinal Study (CHARLS). Int J Epidemiol 43 (1), 61-68. 


\section{Figure Legend}

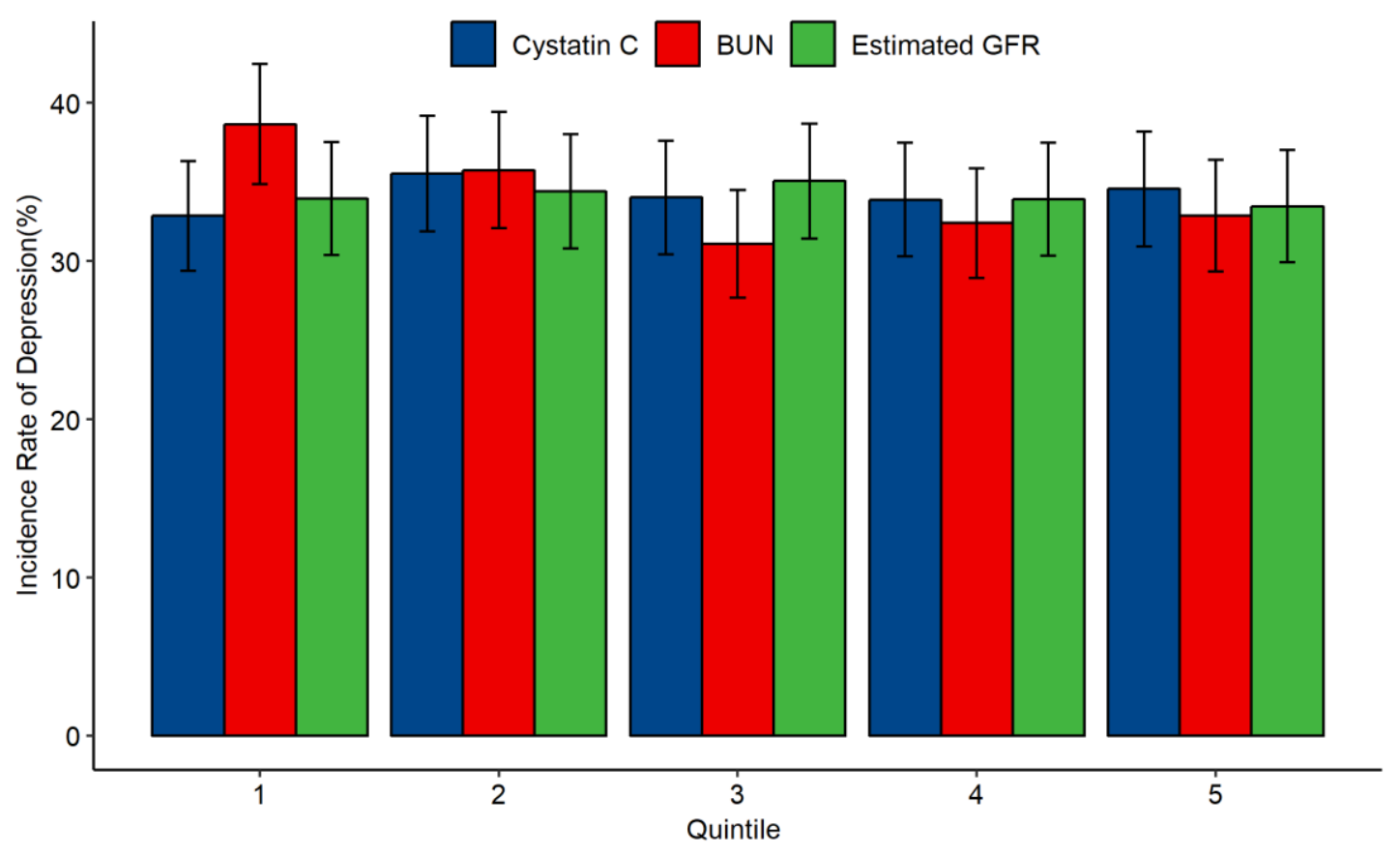

Figure 1. Crude Incidence Rate of Depressive Symptoms According to Quintile of Measures of Renal Function. GFR denotes estimated glomerular filtration rate, and BUN means blood urea nitrogen. 


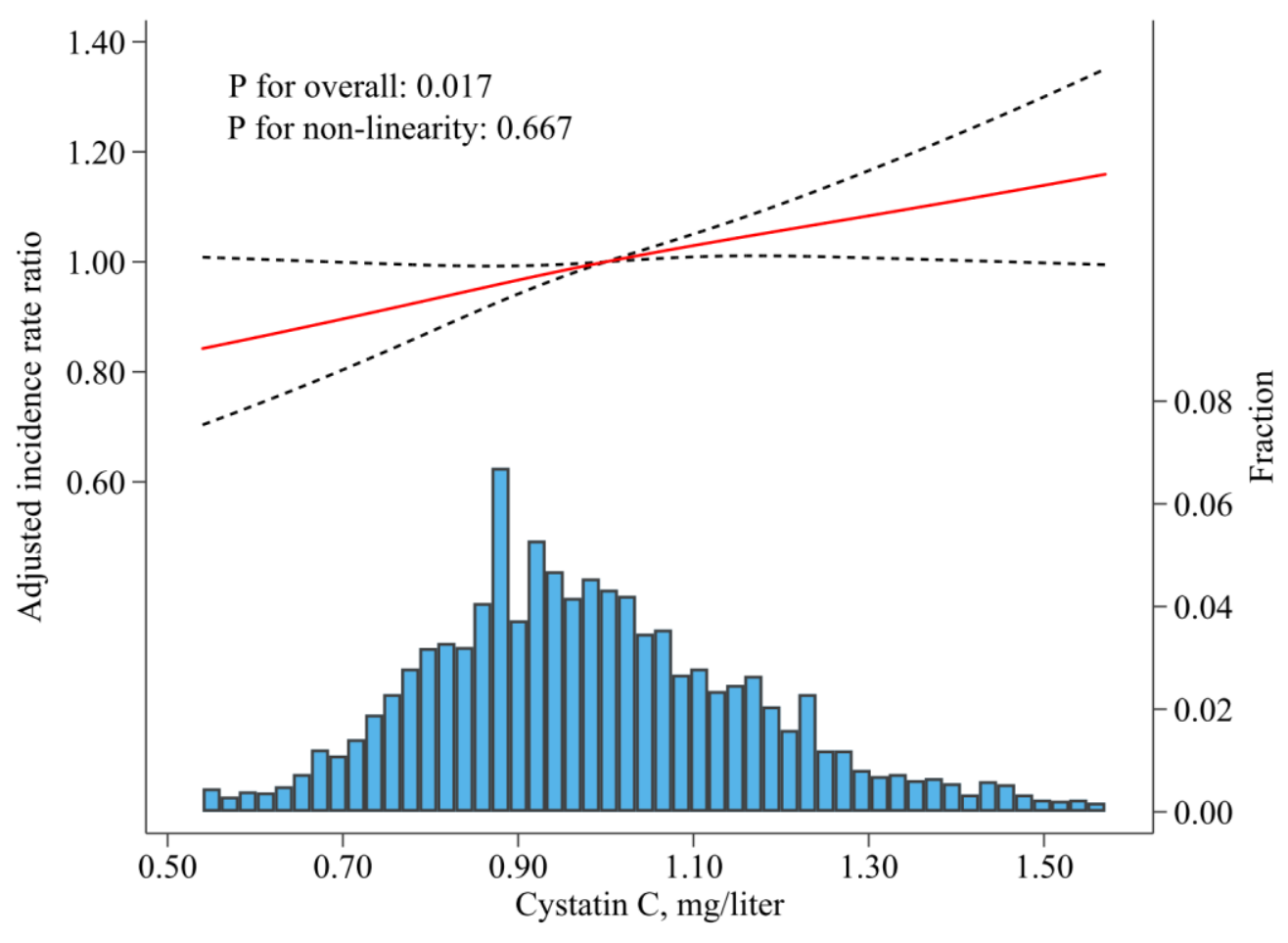

Figure 2. Multivariable Adjusted Incidence Risk Ratio of New-Onset Depressive Symptoms According to Cystatin C levels. Solid-red lines are multivariable-adjusted incidence risk ratio, and dashed lines indicate $95 \%$ confidence intervals derived from restricted cubic spline regression with five knots chosen by Akaike information criterion. Cystatin C of 1.00 $\mathrm{mg} /$ liter was used as the reference because it approximated the median value. The graphs are truncated at the 1st and 99th percentiles. The modified Poisson regression with robust error variance adjusted for age, sex, education, marital status, living area, alcohol use, smoking status, retirement status, social participation, family-based social support, self-reported health, life satisfaction, history of hypertension, diabetes, dyslipidemia, coronary artery heart disease, stroke, and chronic obstructive pulmonary disorder, systolic blood pressure, body-mass index, total, lowdensity, high-density lipoprotein cholesterol levels, log-transformed triglyceride level, logtransformed C-reactive protein level and baseline CES-D score. 
Measurements

Cystatin C

Blood Urea Nitrogen

Estimated GFR
Incidence rate ratio $(95 \% \mathrm{Cl})$

$1.07(1.02-1.11)$

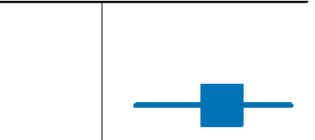

$0.98(0.94-1.02)$

$1.03(0.98-1.07)$

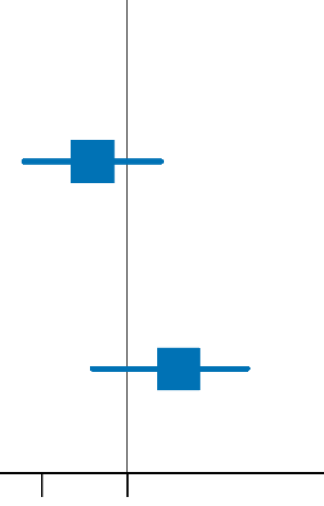

0.951

Figure 3. Association of Renal Function with New-Onset Depressive Symptoms on a Continuous Scale. The incidence risk ratio (IRR) have been adjusted for age, sex, education, marital status, living area, alcohol use, smoking status, retirement status, social participation, family-based social support, self-reported health, life satisfaction, history of hypertension, diabetes, dyslipidemia, coronary artery heart disease, stroke, and chronic obstructive pulmonary disorder, systolic blood pressure, body-mass index, total, low-density, high-density lipoprotein cholesterol levels, log-transformed triglyceride level, log-transformed C-reactive protein level and baseline CES-D score. 


\section{Tables}

Table 1. Baseline Characteristics of Middle-aged Participants in the China Health and Retirement Longitudinal Study, According to Quintiles of Cystatin C

\begin{tabular}{|c|c|c|c|c|c|c|}
\hline Characteristic & $\begin{array}{l}\text { Quintile 1 } \\
(\leq 0.82)\end{array}$ & $\begin{array}{c}\text { Quintile } 2 \\
(0.83-0.92) \\
\end{array}$ & $\begin{array}{c}\text { Quintile } 3 \\
(0.93-1.01) \\
\end{array}$ & $\begin{array}{c}\text { Quintile } 4 \\
(1.02-1.14) \\
\end{array}$ & $\begin{array}{c}\text { Quintile } 5 \\
(\geq 1.15) \\
\end{array}$ & $\begin{array}{l}P \text { for } \\
\text { trend }\end{array}$ \\
\hline No. of participants & 1047 & 1022 & 1020 & 1018 & 1004 & \\
\hline Age - years & $54.4 \pm 7.6$ & $56.2 \pm 7.9$ & $58.1 \pm 8.1$ & $60.9 \pm 8.7$ & $66.0 \pm 9.2$ & $<0.001$ \\
\hline Median $(\min -\max )$ & $53(45-82)$ & $55(45-82)$ & $57(45-83)$ & $60(45-88)$ & $67(45-93)$ & \\
\hline Male sex -no. $(\%)$ & $352(33.6)$ & $437(42.8)$ & $541(53.0)$ & $586(57.6)$ & $675(67.2)$ & $<0.001$ \\
\hline Area urban -no. $(\%)$ & $451(43.1)$ & $395(38.6)$ & $401(39.3)$ & $393(38.6)$ & $389(38.7)$ & 0.068 \\
\hline Education -no. (\%) & & & & & & $<0.001$ \\
\hline No formal education & $392(37.4)$ & $421(41.2)$ & $428(42.0)$ & $451(44.3)$ & $477(47.5)$ & \\
\hline Primary school & $211(20.2)$ & $203(19.9)$ & $237(23.2)$ & $233(22.9)$ & $264(26.3)$ & \\
\hline Middle school or above & $444(42.4)$ & $398(38.9)$ & $355(34.8)$ & $334(32.8)$ & $263(26.2)$ & \\
\hline Smoking status - no. $(\%)$ & & & & & & $<0.001$ \\
\hline Never & $762(72.8)$ & $683(66.8)$ & $582(57.1)$ & $519(51.0)$ & $475(47.3)$ & \\
\hline Former & $66(6.3)$ & $69(6.8)$ & $91(8.9)$ & $107(10.5)$ & $112(11.2)$ & \\
\hline Current & $219(20.9)$ & $270(26.4)$ & $347(34.0)$ & $392(38.5)$ & $417(41.5)$ & \\
\hline Alcohol use -no. $(\%)$ & & & & & & $<0.001$ \\
\hline Never & $742(70.9)$ & $686(67.1)$ & $631(61.9)$ & $627(61.6)$ & $638(63.5)$ & \\
\hline$<1$ times/month & $72(6.9)$ & $71(6.9)$ & $84(8.2)$ & $84(8.3)$ & $82(8.2)$ & \\
\hline$\geq 1$ times/month & $233(22.3)$ & $265(25.9)$ & $305(29.9)$ & $307(30.2)$ & $284(28.3)$ & \\
\hline Married —no. $(\%)$ & $978(93.4)$ & $936(91.6)$ & $933(91.5)$ & $910(89.4)$ & $840(83.7)$ & $<0.001$ \\
\hline Retired-no. $(\%)^{*}$ & $221(21.2)$ & $211(20.7)$ & $226(22.2)$ & $291(28.6)$ & $371(37.0)$ & $<0.001$ \\
\hline Social participation-no. $(\%)$ & $573(54.7)$ & $507(49.6)$ & $496(48.6)$ & $471(46.3)$ & $501(49.9)$ & 0.008 \\
\hline \multicolumn{7}{|l|}{ Family-based social support } \\
\hline Network size $-\log$ & $1.2 \pm 0.5$ & $1.2 \pm 0.5$ & $1.2 \pm 0.5$ & $1.1 \pm 0.5$ & $1.1 \pm 0.6$ & $<0.001$ \\
\hline Receiving help-no. $(\%)^{*}$ & $328(31.3)$ & $367(35.9)$ & $363(35.6)$ & $416(40.9)$ & $488(48.6)$ & $<0.001$ \\
\hline Providing help-no. $(\%)^{*}$ & $474(45.3)$ & $418(40.9)$ & $404(39.6)$ & $370(36.3)$ & $319(31.8)$ & $<0.001$ \\
\hline Self-reported health—no. $(\%)^{*}$ & & & & & & 0.002 \\
\hline Very good/good & $323(30.9)$ & $297(29.1)$ & $283(27.7)$ & $276(27.1)$ & $230(22.9)$ & \\
\hline Fair & $556(53.1)$ & $536(52.4)$ & $536(52.5)$ & $554(54.4)$ & $550(54.8)$ & \\
\hline Poor/very poor & $168(16.0)$ & $189(18.5)$ & $201(19.7)$ & $188(18.5)$ & $223(22.2)$ & \\
\hline Life satisfaction-no. $(\%)^{*}$ & & & & & & 0.493 \\
\hline Completely/very satisfied & $246(25.0)$ & $235(24.9)$ & $248(26.6)$ & $258(27.6)$ & $234(26.1)$ & \\
\hline Somewhat satisfied & $649(66.0)$ & $616(65.3)$ & $596(64.0)$ & $592(63.4)$ & $575(64.2)$ & \\
\hline Not very/not at all satisfied & $88(9.0)$ & $93(9.9)$ & $87(9.3)$ & $84(9.0)$ & $87(9.7)$ & \\
\hline History of hypertension -no. (\%) & $214(20.4)$ & $212(20.7)$ & $234(22.9)$ & $249(24.5)$ & $287(28.6)$ & $<0.001$ \\
\hline History of diabetes —no. (\%) & $72(6.9)$ & $49(4.8)$ & $57(5.6)$ & $39(3.8)$ & $50(5.0)$ & 0.029 \\
\hline History of dyslipidemia —no. (\%) & $98(9.4)$ & $94(9.2)$ & $103(10.1)$ & $78(7.7)$ & $71(7.1)$ & 0.029 \\
\hline History of CHD -no. (\%) & $51(4.9)$ & $74(7.2)$ & $90(8.8)$ & $100(9.8)$ & $115(11.5)$ & $<0.001$ \\
\hline
\end{tabular}




\begin{tabular}{|c|c|c|c|c|c|c|}
\hline listory of stroke - no. $(\%)$ & & $(1.0)$ & & & & 0.022 \\
\hline Iistory of COPD —no. (\%) & $0(8.6)$ & $82(8.0)$ & $107(10.5)$ & $121(11.9)$ & $136(13.5)$ & $<0.001$ \\
\hline mass index $-\mathrm{kg} / \mathrm{m}^{2 *}$ & $24.4 \pm 3.9$ & $23.8 \pm 3.6$ & $23.6 \pm 3.7$ & $23.5 \pm 4.1$ & $22.9 \pm 4.0$ & $<0.001$ \\
\hline oressure $-\mathrm{mm} \mathrm{Hg} *$ & $127.4 \pm 19.5$ & $128.9 \pm 20.5$ & $130.0 \pm 21.0$ & $132.5 \pm 22.1$ & $134.1 \pm 23.0$ & $<0.001$ \\
\hline pressure $-\mathrm{mm} \mathrm{Hg}^{*}$ & $75.9 \pm 11.4$ & $75.9 \pm 11.8$ & $75.8 \pm 12.0$ & $76.5 \pm 12.4$ & \pm 12.5 & 0.289 \\
\hline$-\mathrm{mg} / \mathrm{dl}$ & $199.7 \pm 40.7$ & $192.2 \pm 36.3$ & $192.4 \pm 37.8$ & $191.2 \pm 37.6$ & 188. & $<0.001$ \\
\hline$/ \mathrm{dl}^{*}$ & $116.3 \pm 35.7$ & $117.4 \pm 3$ & $116.8 \pm ?$ & 116.3 & 114 & 0.130 \\
\hline $1-\mathrm{mg} / \mathrm{dl}^{*}$ & $48.4 \pm 14.3$ & $51.5 \pm 14.6$ & $51.1 \pm 15.4$ & $51.0 \pm 15.1$ & $51.6 \pm 15.7$ & $<0.001$ \\
\hline$-\log$ & $4.9 \pm 0.6$ & $4.7 \pm 0.5$ & $4.7 \pm 0.6$ & $4.7 \pm 0.5$ & $4.6 \pm 0.5$ & $<0.001$ \\
\hline lucose $-\mathrm{mg} / \mathrm{c}$ & $16.3 \pm 49.0$ & $109.0 \pm 32.8$ & $108.3 \pm 29.9$ & $107.6 \pm 26.7$ & 106. & $<0.001$ \\
\hline in $-\log$ & $0.0 \pm 1.0$ & $0.0 \pm 1.0$ & $0.1 \pm 0.9$ & 0.2 & 0.4 & $<0.001$ \\
\hline$-\mathrm{mg} / \mathrm{liter}$ & $0.7 \pm 0.1$ & $0.9 \pm 0.0$ & $1.0 \pm 0.0$ & $1.1 \pm 0.0$ & $1.3 \pm 0.2$ & $<0.001$ \\
\hline$-\mathrm{mg} / \mathrm{dl}$ & $0.7 \pm 0.1$ & $0.7 \pm 0.1$ & $0.8 \pm 0.1$ & $0.8 \pm 0.1$ & 0.2 & $<0.001$ \\
\hline $3 \mathrm{UN}-\mathrm{mg} / \mathrm{dl}$ & $14.5 \pm 4.0$ & $14.9 \pm 3.8$ & $15.6 \pm 4.0$ & $16.3 \pm 4.5$ & $17.1 \pm 4.8$ & $<0.001$ \\
\hline Estimated GFR $-\mathrm{m}$ & $100.7 \pm 11.5$ & $97.5 \pm 10.6$ & $94.8 \pm 10.9$ & $90.0 \pm 11.3$ & $82.6 \pm 12.5$ & $<0.001$ \\
\hline aseline CES-D score & $5.1 \pm 3.2$ & $5.4 \pm 3.4$ & $5.2 \pm 3.4$ & $5.3 \pm 3.3$ & $5.3 \pm 3.3$ & 0.262 \\
\hline
\end{tabular}

Data are given as mean \pm SD value or number (percentage) of participants.

* Missing data: retired $(n=1)$, receiving help $(n=1)$, providing help $(n=1)$, life satisfaction $(n=407)$, body-mass index $(n=581)$, systolic blood pressure $(n=583)$, diastolic blood pressure $(n=582)$, LDL cholesterol $(n=1)$, HDL cholesterol $(n=1)$, Fasting glucose $(n=1)$.

CHD denotes coronary artery heart disease, COPD denotes chronic obstructive pulmonary disease, LDL denotes low-density lipoprotein, HDL denotes high-density lipoprotein, BUN denotes blood urea nitrogen, GFR denotes glomerular filtration rate, and CES-D denotes Center for Epidemiologic Studies Depression Scale.

$\mathrm{C}$-reactive protein was measured in milligrams per liter. 
Table 2. Risk of Depression According to Measures of Renal Function among Middle-aged Participants in the China Health and Retirement Longitudinal Study

\begin{tabular}{|c|c|c|c|c|c|}
\hline & Quintile 1 & Quintile 2 & Quintile 3 & Quintile 4 & Quintile 5 \\
\hline \multicolumn{6}{|c|}{ Cystatin C - mg/liter } \\
\hline Range of values & $\leq 0.82$ & $0.83-0.92$ & $0.93-1.01$ & $1.02-1.14$ & $\geq 1.15$ \\
\hline No. at risk & 1047 & 1022 & 1020 & 1018 & 1004 \\
\hline No. of depression & 344 & 363 & 347 & 345 & 347 \\
\hline \multicolumn{6}{|c|}{ Incidence rate ratio $(95 \% \mathrm{CI})$} \\
\hline Unadjusted & 1.00 & $1.08(0.96-1.22)$ & $1.04(0.92-1.17)$ & $1.03(0.91-1.17)$ & $1.05(0.93-1.19)$ \\
\hline Full adjusted $\dagger$ & 1.00 & $1.08(0.96-1.22)$ & $1.09(0.96-1.23)$ & $1.11(0.97-1.26)$ & $1.18(1.03-1.35)$ \\
\hline \multicolumn{6}{|c|}{ Blood Urea Nitrogen (BUB) - mg/dl } \\
\hline Range of values & $\leq 12.0$ & $12.1-14.12$ & $14.13-16.16$ & $16.17-19.1$ & $\geq 19.2$ \\
\hline No. at risk & 1032 & 1015 & 1023 & 1031 & 1010 \\
\hline No. of depression & 399 & 363 & 318 & 334 & 332 \\
\hline \multicolumn{6}{|c|}{ Incidence rate ratio $(95 \% \mathrm{CI})$} \\
\hline Unadjusted & 1.00 & $0.93(0.83-1.04)$ & $0.80(0.71-0.91)$ & $0.84(0.75-0.94)$ & $0.85(0.76-0.96)$ \\
\hline Full adjusted $\dagger$ & 1.00 & $0.96(0.86-1.08)$ & $0.86(0.76-0.97)$ & $0.91(0.81-1.03)$ & $0.92(0.82-1.04)$ \\
\hline \multicolumn{6}{|c|}{ Estimated GFR - $\mathrm{ml} / \mathrm{min} / 1.73 \mathrm{~m}^{2}$} \\
\hline Range of values & $\geq 104.43$ & $98.36-104.42$ & $91.58-98.35$ & $82.72-91.57$ & $\leq 82.71$ \\
\hline No. at risk & 1025 & 1020 & 1024 & 1020 & 1022 \\
\hline No. of depression & 348 & 351 & 359 & 346 & 342 \\
\hline \multicolumn{6}{|c|}{ Incidence rate ratio $(95 \% \mathrm{CI})$} \\
\hline Unadjusted & $1.01(0.90-1.15)$ & $1.03(0.91-1.16)$ & $1.05(0.93-1.18)$ & $1.01(0.90-1.14)$ & 1.00 \\
\hline Full adjusted $\uparrow$ & $1.10(0.96-1.26)$ & $1.15(1.01-1.31)$ & $1.13(0.99-1.28)$ & $1.03(0.91-1.16)$ & 1.00 \\
\hline
\end{tabular}

$\dagger$ Adjusted for age, sex, education, marital status, living area, alcohol use, smoking status, retirement status, social participation, family-based social support, self-reported health, life satisfaction, history of hypertension, diabetes, dyslipidemia, coronary artery heart disease, stroke, and chronic obstructive pulmonary disorder, systolic blood pressure, body-mass index, total, low-density, high-density lipoprotein cholesterol levels, log-transformed triglyceride level, log-transformed C-reactive protein level and baseline CES-D score. 


\section{Supplementary Materials}

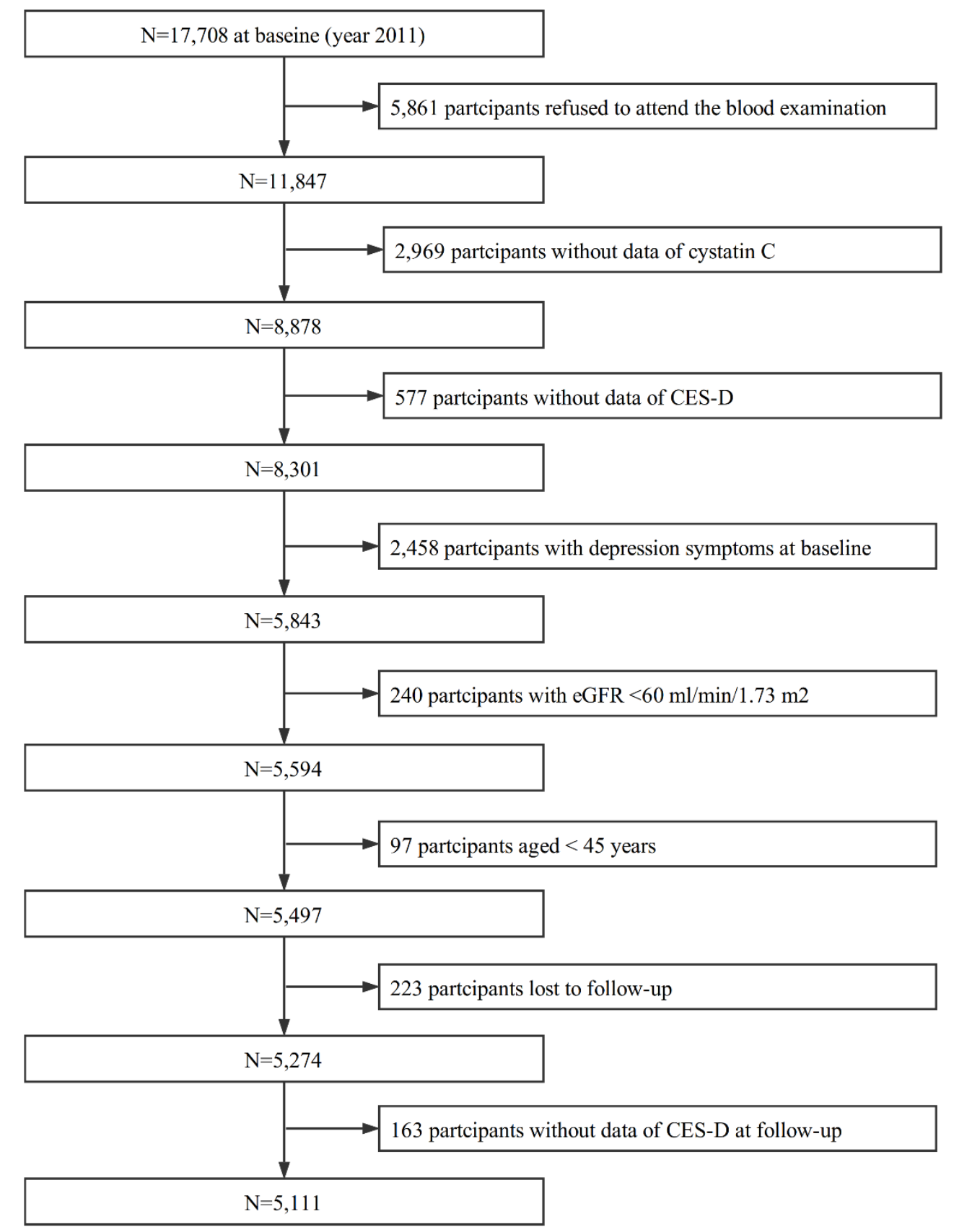

Figure S1. Flow Diagram of the Participants Selection from the China Health and Retirement Longitudinal Study. 
Table S1. Incidence rate ratio (95\% CI) of Depression According to Measures of Renal Function among Middle-aged Participants in the China Health and Retirement Longitudinal Study $(\mathrm{n}=4128)$

\begin{tabular}{lcccccc}
\hline & Quintile 1 & Quintile 2 & Quintile 3 & Quintile 4 & Quintile 5 & 1-SD change \\
\hline $\begin{array}{l}\text { Cystatin C - mg/liter } \\
\quad \text { Full adjusted } \dagger\end{array}$ & 1.00 & $1.10(0.96-1.26)$ & $1.04(0.90-1.20)$ & $1.13(0.97-1.30)$ & $1.20(1.02-1.40)$ & $1.06(1.02-1.12)$ \\
Blood Urea Nitrogen (BUB) - mg/dl & & & & & \\
$\quad \begin{array}{l}\text { Full adjusted } \dagger \\
\quad \text { Estimated GFR }-\mathrm{ml} / \mathrm{min} / 1.73 \mathrm{~m}^{2}\end{array}$ & $0.96(0.84-1.10)$ & $0.91(0.80-1.04)$ & $0.94(0.82-1.08)$ & $0.95(0.83-1.09)$ & $0.99(0.95-1.04)$ \\
Full adjusted $\dagger$ & $1.17(0.99-1.37)$ & $1.19(1.02-1.39)$ & $1.19(1.03-1.38)$ & $1.02(0.88-1.18)$ & 1.00 & $1.06(1.00-1.11)$ \\
\hline
\end{tabular}

Data are incidence rate ratio and the $95 \% \mathrm{CI}$.

$\dagger$ Adjusted for age, sex, education, marital status, living area, alcohol use, smoking status, retirement status, social participation, family-based social support, self-reported health, life satisfaction, history of hypertension, diabetes, dyslipidemia, coronary artery heart disease, stroke, and chronic obstructive pulmonary disorder, systolic blood pressure, body-mass index, total, low-density, high-density lipoprotein cholesterol levels, log-transformed triglyceride level, log-transformed C-reactive protein level and baseline CES-D score. 
